Discrete Comput Geom 32:373-382 (2004)

DOI: $10.1007 / \mathrm{s} 00454-004-2906-4$

\title{
Directed Trees in a String, Real Polynomials with Triple Roots, and Chain Mails*
}

\author{
Dmitry N. Kozlov \\ Department of Mathematics, Royal Institute of Technology, \\ Stockholm S-100 44, Sweden \\ kozlov@math.kth.se
}

\begin{abstract}
This paper starts with an observation that two infinite series of simplicial complexes, which a priori do not seem to have anything to do with each other, have the same homotopy type. One series consists of the complexes of directed forests on a double directed string, while the other one consists of Shapiro-Welker models for the spaces of hyperbolic polynomials with a triple root.

We explain this coincidence in the more general context by finding an explicit homotopy equivalence between complexes of directed forests on a double directed tree, and doubly disconnecting complexes of a tree.
\end{abstract}

\section{Observation}

When the explicit determination of the homotopy type of families of combinatorially defined simplicial complexes is performed, it sometimes happens that the answers coincide. In such a case two natural problems arise immediately. First, one would like to have an explicit map between these complexes, which would provide a homotopy equivalence. Second, one may ask for new terminology and a new theorem, which would put this particular result in a more general context. This paper is an example of an analysis of a situation like this.

\subsection{Complexes of Directed Forests}

We start by describing the two families of simplicial complexes, for which the computations yield the same answer.

* This research was supported by a Research Grant of the Swiss Natural Science Foundation. 
Definition 1.1 [4], [9]. Let $G$ be an arbitrary directed graph. $\Delta(G)$ is the simplicial complex $\Delta(G)$ constructed as follows: the vertices of $\Delta(G)$ are given by the edges of $G$ and faces are all directed forests which are subgraphs of $G$.

The complexes of directed forests in a given directed graph were introduced in [4] following the suggestion of R. Stanley. It was proved in [4] that $\Delta(G)$ is shellable (hence homotopy equivalent to a wedge of spheres) when $G$ has a complete sink. Furthermore, the homotopy types of complexes $\Delta(G)$ were computed for several natural families of graphs $G$. The special case, which is of particular importance for this paper is when $G$ is a double directed string.

Definition 1.2 [4]. Let $n$ be a nonnegative integer. A double directed string on $n+1$ vertices is the directed graph, denoted $L_{n}$, which is defined by $V\left(L_{n}\right)=[n+1]$, and $E\left(L_{n}\right)=\{(i \rightarrow i+1),(i+1 \rightarrow i) \mid i \in[n]\}$.

The following proposition was also proved in [4].

\section{Proposition 1.3.}

$$
\Delta\left(L_{n}\right) \simeq \begin{cases}S^{2 k-1}, & \text { if } n=3 k \\ S^{2 k}, & \text { if } n=3 k+1 \\ \text { a point, } & \text { if } n=3 k+2\end{cases}
$$

\subsection{Spaces of Monic Hyperbolic Polynomials with Multiple Roots}

We now turn to the second family of simplicial complexes. The space of monic hyperbolic polynomials in one variable of degree $n$, which we denote by Hyp ${ }^{n}$, is naturally stratified by fixing the multiplicities of roots (in our terminology a polynomial is called hyperbolic if all of its roots are real).

These strata are indexed by number partitions which refine $n$, and for a partition $\lambda=\left(\pi_{1}, \ldots, \pi_{t}\right)$ we let $\widehat{\operatorname{Hyp}_{\lambda}^{n}}$ denote the one-point compactification of the set of all polynomials of the form $\left(x-r_{1}\right)^{\pi_{1}} \cdots\left(x-r_{t}\right)^{\pi_{t}}$ (note that we do not require that $r_{1}, \ldots, r_{t}$ are distinct numbers).

To fix the multiplicities of roots is the same as fixing a number partition $\lambda \vdash n$. While one can think of a number partition $\lambda=\left(\pi_{1}, \ldots, \pi_{t}\right)$ simply as a set of numbers, such that $\pi_{1}+\cdots+\pi_{t}=n$, we use the notation $\left[\pi_{1}, \ldots, \pi_{t}\right]$ to denote the ordered $t$-tuple of these numbers. In such a situation, $\left[\pi_{1}, \ldots, \pi_{t}\right]$ is called a composition of $n$. By forgetting the order of the numbers in the composition we get a number partition which is called the type of this composition. For both a partition and a composition, we call the number of its parts the length, and denote it by $l(\lambda)$, resp. $l\left(\left[\pi_{1}, \ldots, \pi_{t}\right]\right)$.

The set of all compositions of $n$ is partially ordered by refinement. Namely, let $x=$ $\left[\alpha_{1}, \ldots, \alpha_{l(x)}\right]$ and $y=\left[\beta_{1}, \ldots, \beta_{l(y)}\right]$ be two compositions of $n$, we say that $x \leq y$ if and only if $\alpha_{j}=\beta_{i_{j-1}+1}+\cdots+\beta_{i_{j}}$, for $1 \leq j \leq l(x)$, and some $0=i_{0}<i_{1}<\cdots<$ $i_{l(x)}=l(y)$. Since $\beta_{i}>0$, for $i=1, \ldots, l(y)$, the indices $i_{1}, \ldots, i_{l(x)-1}$ are uniquely defined. 
Given a number partition $\lambda=\left(\pi_{1}, \ldots, \pi_{t}\right)$ of $n$, we define $D_{\lambda}$ to be the poset consisting of all compositions of $n$ which are less than or equal to some composition of $n$ of type $\lambda$. Thus, the number of maximal elements of $D_{\lambda}$ is equal to the number of different ways to impose an order on the numbers $\pi_{1}, \ldots, \pi_{t}$. Note that $D_{\lambda}$ has a minimal element, the composition consisting of just the number $n$, and it is easy to see that $D_{\lambda} \cup\{\hat{1}\}$ is a lattice, where $\hat{1}$ is an artificially added maximal element.

Since the lower intervals of $D_{\lambda}$ are Boolean algebras, and $D_{\lambda}$ itself is a meetsemilattice, there exists a unique simplicial complex, which we denote by $\delta_{\lambda}$, such that $D_{\lambda}$ is the face poset of $\delta_{\lambda}$, i.e., the elements of $D_{\lambda}$ and the simplices of $\delta_{\lambda}$ are in bijection, and the partial order relation on $D_{\lambda}$ corresponds under this bijection to the inclusions of simplices of $\delta_{\lambda}$.

As the following theorem, proved in [8], shows, the simplicial complex $\delta_{\lambda}$ provides a combinatorial model for the stratum $\widehat{\operatorname{Hyp}_{\lambda}^{n}}$.

Theorem 1.4 [8, Theorem 3.5(a)]. Let $\lambda$ be a number partition of $n$, then the onepoint compactification of the strata indexed by $\lambda, \widehat{H y p_{\lambda}^{n}}$, is homeomorphic to the double suspension of the simplicial complex $\delta_{\lambda}$.

Complete descriptions of the homotopy type of the simplicial complexes $\delta_{\lambda}$ were obtained for several classes of number partitions $\lambda$, see [5], [6], and [8]. In particular the following is well known.

Proposition 1.5 [8, Corollary 3.10], [5, Proposition 3.4(a)]. For $\lambda=\left(k, 1^{t}\right)$, where $k \geq 2, t \geq 0$, we have

$$
\delta_{\lambda} \simeq \begin{cases}S^{2 m-1}, & \text { if } t=k m, \quad \text { for some } m \in \mathbb{Z} ; \\ S^{2 m}, & \text { if } t=k m+1, \text { for some } m \in \mathbb{Z} ; \\ \text { point, } & \text { otherwise. }\end{cases}
$$

By comparing Propositions 1.3 and 1.5, we get that

$$
\delta_{\left(3,1^{t}\right)} \simeq \Delta\left(L_{t}\right) .
$$

In the next section we provide an explicit homotopy equivalence between $\delta_{\left(3,1^{t}\right)}$ and $\Delta\left(L_{t}\right)$, while we state and prove a more general theorem in Section 3.

\section{Explicit Homotopy Equivalence}

\subsection{Further Descriptions of the Simplicial Complexes $\delta_{\left(3,1^{t}\right)}$ and $\Delta\left(L_{t}\right)$}

Let us note an alternative description of the simplicial complex $\delta_{\left(3,1^{t}\right)}$. We take as simplices all subsets $\sigma$ of $\{1, \ldots, t+2\}$, for which there exist $1 \leq i \leq t+1$, such that $i, i+1 \notin \sigma$; in other words, the maximal simplices are precisely all sets $\{1, \ldots, t+2\} \backslash\{i, i+1\}$, for $i=1, \ldots, t+1$. Please note that we step a bit away from the usual conventions of the simplicial complexes, in that in our description it is not 


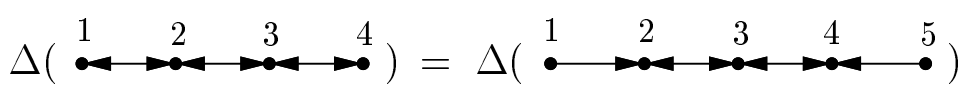

Fig. 1. The case $\mathrm{t}=3$.

necessary that all 1-element subsets of $\{1, \ldots, t+2\}$ are simplices. For example, $\delta_{(3)}$ is simply empty, while $\delta_{(3,1)}$ has only two vertices.

Also $\Delta\left(L_{t}\right)$ has other descriptions, two of which we list next.

Description 1 (via forbidden patterns). $\Delta\left(L_{t}\right)$ is the simplicial complex, with the set of vertices being the set $\{1, \ldots, 2 t\}$, where we take as simplices all subsets $\sigma \subseteq\{1, \ldots, 2 t\}$, such that $\{i, i+1\} \nsubseteq \sigma$, for any $i=1, \ldots, 2 t-1$. These simplicial complexes have appeared in various guises (for example, in [2] they were called sparse complexes).

With this description at hand it is obvious that the simplicial complex $\Delta\left(L_{t}\right)$ is isomorphic to $\Delta\left(L_{t}^{\prime}\right)$, where $L_{t}^{\prime}$ is the directed graph on $t+2$ vertices, $V\left(L_{t}^{\prime}\right)=\{1, \ldots, t+$ $2\}$, given by

$$
E\left(L_{t}^{\prime}\right)=\{(1 \rightarrow 2),(t+2 \rightarrow t+1)\} \cup\{(i \rightarrow i+1),(i+1 \rightarrow i) \mid i=2, \ldots, t\},
$$

see Fig. 1.

More generally, one has the following definition.

Definition 2.1. For an arbitrary graph $\Gamma$ we can define the independence simplicial complex $\mathcal{I}(\Gamma)$ as follows. The set of vertices of $\mathcal{I}(\Gamma)$ is equal to the set of vertices of $\Gamma$. The subset $\sigma \subseteq \mathcal{I}(\Gamma)$ is a simplex if and only if the vertices in $\sigma$ can be colored with the same color.

Note that we use the usual requirement, that no two vertices of the same color are connected by an edge. In the graph theory literature, sets of vertices which can be colored with the same color are traditionally called independent sets, which is why we chose this name for the complexes $\mathcal{I}(\Gamma)$. The condition for being a simplex in Definition 2.1 could be reformulated slightly differently, namely, if $\sigma$ is a simplex of $\mathcal{I}(\Gamma)$, and $(x, y)$ is an edge of $\Gamma$, then either $x \notin \sigma$ or $y \notin \sigma$.

Description 2 (via order complexes). Recall that to any partially ordered set (poset for short) one can associate a simplicial complex whose vertex set is the set of the elements of the poset, and whose set of simplices consists of all the completely ordered subsets of elements of the poset (these ordered subsets are also known as chains). This simplicial complex is called the order complex of the poset, see [1]. Connecting this definition to our situation, one can see that $\Delta\left(L_{t}\right)$ is isomorphic to the order complex of the poset $P_{t}$ (Fig. 2), given by:

- the set of the elements of $P_{t}$ is $\{1, \ldots, 2 t\}$;

- the partial order on $P_{t}$ is given by $x>y$ if and only if $|x-y| \geq 2$.

Note that it is rather unusual for a graph $G$ to be such that there exists a poset $P$, such that $\Delta(G)=\Delta(P)$. For example, a careful check should convince the reader that no such poset $P$ exists for the graph of Fig. 3. 


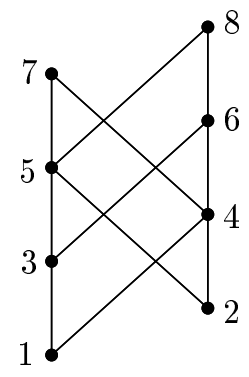

Fig. 2. The poset $P_{4}$.

\subsection{The Explicit Homotopy Equivalence}

We are now in the position to define the map which shall give an explicit homotopy equivalence between $\Delta\left(L_{t}\right)$ and $\delta_{\left(3,1^{t}\right)}$. Namely, let $\varphi$ be the simplicial map defined by

$$
\varphi: \begin{array}{ccc}
\Delta\left(L_{t}\right) & \longrightarrow & \delta_{\left(3,1^{t}\right)}, \\
(x \rightarrow y) & \longmapsto & x,
\end{array}
$$

here we use the description of $\delta_{\left(3,1^{t}\right)}$ given in Section 2.1, and the description of $\Delta\left(L_{t}\right)$ as being isomorphic to $\Delta\left(L_{t}^{\prime}\right)$; in particular, the vertex sets of $L_{t}^{\prime}$ and $\delta_{\left(3,1^{t}\right)}$ are both denoted by $[t+2]$.

Theorem 2.2. The induced map of topological spaces $\varphi: \Delta\left(L_{t}\right) \longrightarrow \delta_{\left(3,1^{t}\right)}$ is a homotopy equivalence.

It is not difficult to show that $\varphi$ is well-defined and prove Theorem 2.2. However, we do not do that here, as it follows from the more general Theorem 3.2 in the next section.

\subsection{Chain Mails}

To conclude this section we give yet another interpretation of the simplicial complexes $\Delta\left(L_{t}\right)$ and $\delta_{\left(3,1^{t}\right)}$, which allows us to view the map $\varphi$ in a somewhat different light.

Consider $t+2$ unit rings arranged as shown in Fig. 4.

If we identify the rings with the vertices of $\delta_{\left(3,1^{t}\right)}$, then the complex $\delta_{\left(3,1^{t}\right)}$ is identified with the simplicial complex on the set of rings, in which the simplices are all collections of rings with at least two missing rings in a row.

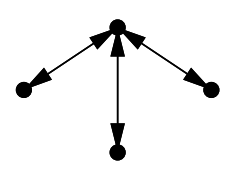

Fig. 3. A graph $G$ without a poset $P$ such that $\Delta(G)=\Delta(P)$. 


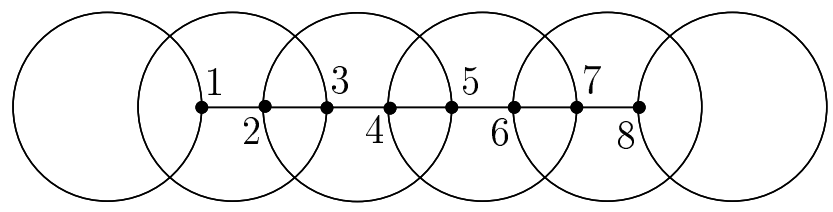

Fig. 4. Ring arrangement for $t=4$.

We now associate a graph $\Gamma$ to this string of rings as follows. We take a vertex on each small (smaller than $\pi$ ) arc of the ring, which is cut out by another ring, except for the two small arcs on the ends. Then we connect each pair of vertices, where an edge can be drawn without intersecting the rings; that is, we connect by an edge each pair of vertices which see each other without having to look through the rings. Clearly, we obtain a string with $2 t$ vertices, and it is immediate that $\mathcal{I}(\Gamma)=\Delta\left(L_{t}\right)$.

Using the description of $\Delta\left(L_{t}\right)$ via forbidden patterns, we are now able to see our map $\varphi: \mathcal{I}(\Gamma) \longrightarrow \delta_{\left(3,1^{t}\right)}$ as the one which maps each vertex of $\Gamma$ to the ring to which it belongs. This mental image is the main reason for introducing more general structures, which we, following the visual analogy, call chain mails.

In fact, there are various ways to generalize the situation shown in Fig. 4. We choose one which will be sufficient for our purposes.

Definition 2.3. A chain mail is a collection of polygons (more precisely, not necessarily convex disks with polynomial boundary) $R_{1}, \ldots, R_{p}$ on the plane, such that

- for all $i<j, R_{i} \nsubseteq R_{j}$ and $R_{j} \nsubseteq R_{i}$;

- for all $i<j$, if $R_{i} \cap R_{j}$ is nonempty, then $\partial R_{i} \cap \partial R_{j}$ consists of two points and $\left(R_{i} \backslash \partial R_{i}\right) \cap\left(R_{j} \backslash \partial R_{j}\right) \neq \emptyset$;

- each triple intersection $R_{i} \cap R_{j} \cap R_{k}, i<j<k$, is empty.

Note that if $\partial R_{i} \cap \partial R_{j} \neq \emptyset$, then the boundary of each of the PL curves $\partial R_{i}$ and $\partial R_{j}$ is divided into two parts: one part lies inside $R_{j}$, resp. $R_{i}$, while the other one lies outside.

To each chain mail one can associate a graph $\Gamma$ as follows:

- Vertices of $\Gamma$. For each pair $i \neq j, i, j \in\{1, \ldots, p\}$, such that $\partial R_{i} \cap \partial R_{j} \neq \emptyset$, we put one vertex $v$ of the graph $G$ on the part of $\partial R_{i}$ which lies in $R_{j} \backslash \partial R_{j}$ if there exists $k \in\{1, \ldots, p\}, k \neq i, k \neq j$, such that one can connect $v$ with $\partial R_{k}$, with a PL curve, without intersecting any $\partial R_{h}, h \in\{1, \ldots, p\}$, except at the endpoints of the given curves; see Fig. 6 .

- Edges of $\Gamma$. Two vertices are connected by an edge, if they can be connected by a PL curve, which does not intersect $\partial R_{h}$, for all $h \in\{1, \ldots, p\}$, other than at its endpoints; again see Fig. 6.

\section{Homotopy Equivalence in the Context of Doubly Connected Complexes}

In this section we generalize Theorem 2.2, and give a direct proof of this generalization. 


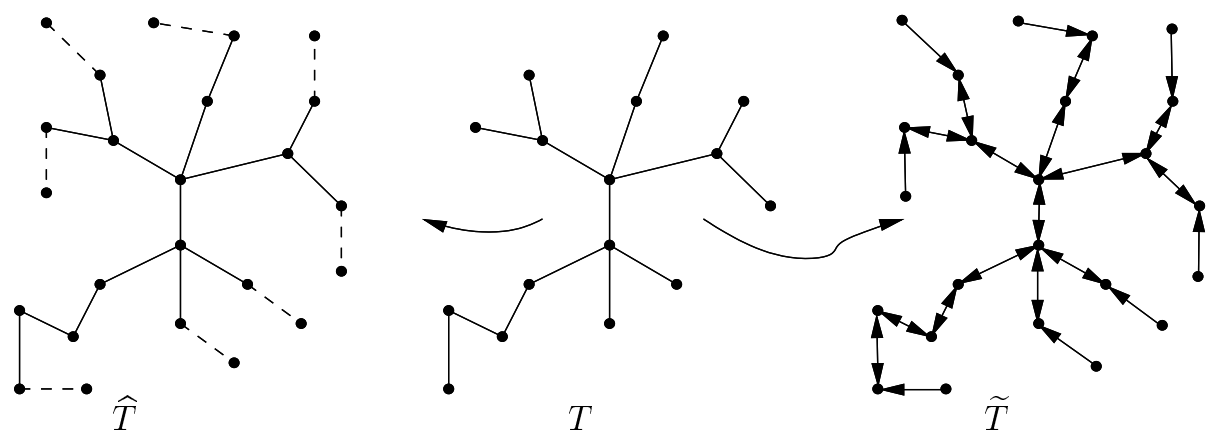

Fig. 5. An example of constructing $\hat{T}$ and $\tilde{T}$.

\section{1. $\quad k$-Fold Disconnecting Complexes of Trees}

First we need some terminology. For any tree $T$ there is a natural way to augment it as follows: for each leaf of $T$ we add one extra vertex, which is a new leaf, and one extra edge, which connects the new leaf with the old one. We call the augmented tree $\widehat{T}$ (Fig. 5). Alternatively, the class of all augmented trees could be described as the class of all trees where each path leading from a branch vertex to a leaf is at least of length 2 .

One can also turn the tree into a directed tree, as one augments it, as follows: all the edges of $T$ are replaced by pairs of edges going in opposite directions, while the new edges are each replaced by just one edge, which is directed to point from the new leaf. We denote the thus obtained directed tree by $\widetilde{T}$ (Fig. 5).

Definition 3.1. Given a tree $T$. For a positive integer $k$, let $\mathcal{D}_{k}(T)$ be the simplicial complex defined as follows. The vertices of $\mathcal{D}_{k}(T)$ are the vertices of $\widehat{T}$, while $S \subseteq V(\widehat{T})$ is a simplex if for any path $\left(x_{1}, \ldots, x_{t}\right)$ connecting two different leaves of $\widehat{T}$ (note that $t \geq 4$ is necessitated) there exists $1 \leq i \leq t-k+1$, such that $x_{i}, x_{i+1}, \ldots, x_{i+k-1} \notin S$.

We call $\mathcal{D}_{k}(T)$ the $k$-fold disconnecting complex of $T$. When $k=2$ we simply call $\mathcal{D}_{2}(T)$ the doubly disconnecting complex of $T$. Clearly, when $T$ is a string (a tree without branching) with $t$ vertices, the complex $\mathcal{D}_{2}(T)$ is isomorphic to $\delta_{\left(3,1^{t}\right)}$.

It is natural to associate a chain mail to the graph $\widehat{T}$, as is shown in Fig. 6.

For each vertex of $\widehat{T}$ we draw a (not necessarily convex) polygon, so that these polygons form a chain mail, i.e., satisfy conditions of Definition 2.3, and so that two polygons intersect if and only if the corresponding vertices of $\widehat{T}$ are connected by an edge. We rely on the visual clarity of Fig. 6 for elucidation of how such a chain mail looks.

However, we would like to mention one formal way to construct this chain mail. For each vertex of $\widehat{T}$ take the union of the closed halves of the edges adjacent to this vertex (in the standard metric associated to any graph, this is the closed ball of radius $\frac{1}{2}$ centered at this vertex). Take the closed $\varepsilon$-neighborhood of this union, where $\varepsilon$ is small and depends on the specific embedding of $\widehat{T}$ into the plane. The polygons can now be taken as sufficiently fine (again depending on the embedding of $\widehat{T}$ ) PL approximations 


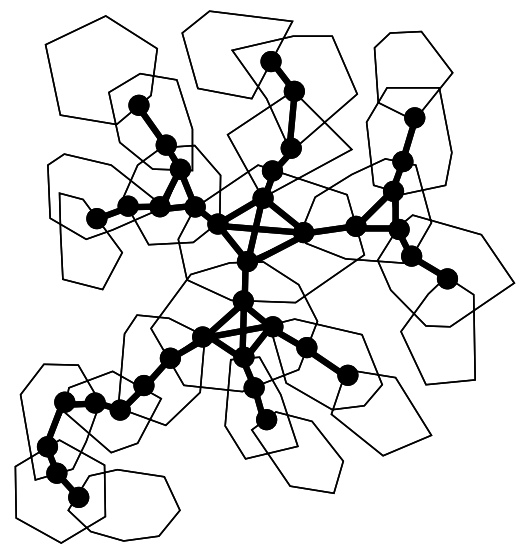

Fig. 6. An example of associating a chain mail to $\hat{T}$.

of these $\varepsilon$-neighborhoods. The graph $\Gamma$ depicted in Fig. 6 is the graph associated to this chain mail, as described in Section 2.3. Note that $\mathcal{I}(\Gamma)=\Delta(\widetilde{T})$.

\subsection{The Main Theorem}

Theorem 3.2. Let $T$ be a tree, then the simplicial complexes $\mathcal{D}_{2}(T)$ and $\Delta(\widetilde{T})$ are homotopy equivalent.

Proof. The map $\varphi: \Delta(\widetilde{T}) \longrightarrow \mathcal{D}_{2}(T)$ is defined analogously to the one in the previous section. One way to describe it is to set $\varphi(x \rightarrow y)=x$. The other way, in terms of chain mails, is to say that each vertex of the graph $\Gamma$ associated to the chain mail is mapped to the boundary of the polygon to which it belongs. Recall that, as mentioned above, $\mathcal{I}(\Gamma)$ is isomorphic to $\Delta(\widetilde{T})$, and we are tacitly talking here about $\varphi: \mathcal{I}(\Gamma) \longrightarrow \mathcal{D}_{2}(T)$.

Claim 1. $\varphi$ is well-defined.

Let $S$ be a simplex of $\Delta(\widetilde{T}), S \neq \emptyset$. We need to show that $\varphi(S)$ has a "2-gap" in each path connecting two leaves. Without loss of generality, we can assume that $S$ is a maximal simplex of $\Delta(\widetilde{T})$.

Assume $\varphi(S)$ is not a simplex of $\mathcal{D}_{2}(T)$, and choose a path $P=\left(x_{1}, \ldots, x_{t}\right)$ in $\mathcal{D}_{2}(T)$ which does not have a 2-gap, again $t \geq 4$. If $\left(x_{1} \rightarrow x_{2}\right) \notin S$ and $\left(x_{2} \rightarrow x_{3}\right) \notin S$, then $x_{1}, x_{2} \notin \varphi(S)$, thus $P$ has a 2-gap, which is a contradiction. In the same way, either $\left(x_{t} \rightarrow x_{t-1}\right) \in S$ or $\left(x_{t-1} \rightarrow x_{t-2}\right) \in S$. So $S$ contains two edges directed to each other, which, by maximality of $S$, means that there must exist $2 \leq i \leq t-2$, such that $\left(x_{i-1} \rightarrow x_{i}\right),\left(x_{i+2} \rightarrow x_{i+1}\right) \in S$, which in turn implies that $x_{i}, x_{i+1} \notin S$. This is again a contradiction to the assumption that $P$ has no 2-gap.

Claim 2. $\varphi$ gives a homotopy equivalence. 


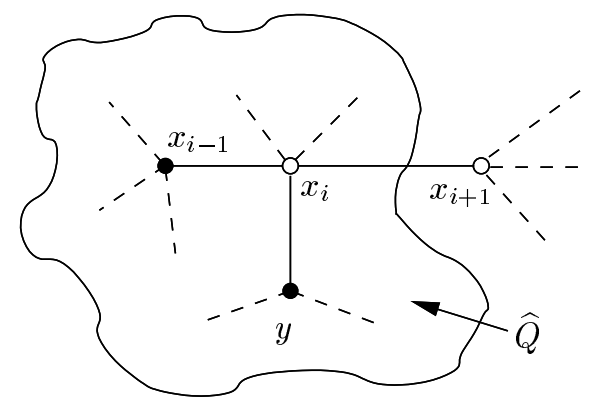

Fig. 7. Construction of $\hat{Q}$.

We shall show that for each (closed) simplex $S \subseteq \mathcal{D}_{2}(T), \varphi^{-1}(S)$ is contractible. In fact, by using induction on the number of vertices of $\widehat{T}$, we shall prove that it is a cone. The theorem then follows by Quillen's lemma, see [7].

Take any path $P=\left(x_{1}, \ldots, x_{t}\right)$ in $\widehat{T}$, such that $P \cap S \neq \emptyset$. Since $P \cap S$ must contain a gap consisting of at least two elements, we can always find an index $i$ (possibly after reversing the indexing of $x_{j}$ 's) such that $2 \leq i \leq t-1, x_{i-1} \in S$ and $x_{i}, x_{i+1} \notin S$.

Obviously, $\left(x_{i-1} \rightarrow x_{i}\right) \in \varphi^{-1}(S)$. If $\varphi^{-1}(S)$ is not a cone with apex at $\left(x_{i-1} \rightarrow x_{i}\right)$, then there must exist some simplex $\sigma$ in $\varphi^{-1}(S)$, such that $\sigma \cup\left\{\left(x_{i-1} \rightarrow x_{i}\right)\right\} \notin \varphi^{-1}(S)$. This means that either $\left(x_{i} \rightarrow x_{i-1}\right) \in \sigma$ or $\left(y \rightarrow x_{i}\right) \in \sigma$, for some $y \neq x_{i-1}$. The first option is ruled out by the fact that $x_{i} \notin S$, furthermore, in the second option we have $y \neq x_{i+1}$, since $x_{i+1} \notin S$. Thus, we conclude that the vertex $x_{i}$ has to have valency of at least 3 , which means $x_{i}$ is an internal vertex (not a leaf) of $T$.

Let $\widehat{Q}$ be the induced subtree of $\widehat{T}$ consisting of all those vertices $v \in \widehat{T}$, which can be reached from $x_{i}$ without passing through $x_{i+1}$; this includes $x_{i}$ itself, see Fig. 7 .

Since the valency of $x_{i}$ in $\widehat{T}$ is at least 3 , its valency in $\widehat{Q}$ is at least 2 , hence each leaf of $\widehat{Q}$ is also a leaf of $\widehat{T}$. Let $Q$ be the tree obtained from $\widehat{Q}$ by deleting all its leaves, and let $\widetilde{Q}$ denote the directed graph associated to $\widehat{Q}$, with the rule of association being the same as before. Let $\varphi_{Q}: \Delta(\widetilde{Q}) \longrightarrow \mathcal{D}_{2}(Q)$ be the restriction of the map $\varphi$.

By the induction assumption, $\varphi_{Q}^{-1}(S \cap \widehat{Q})$ is a cone. We denote its apex by $(x \rightarrow y)$. If $\varphi^{-1}(S)$ is not a cone with apex $(x \rightarrow y)$, then there must exist a simplex $\sigma \in \varphi^{-1}(S)$, such that either $(y \rightarrow x) \in \sigma$ or $(z \rightarrow y) \in \sigma$. By the construction of $\widehat{Q}$, we conclude that the first option is impossible, and in the second option we must have $z=x_{i+1}$. This yields a contradiction, as it implies that $x_{i+1} \in S$.

\section{Further Questions}

As mentioned above, when $T$ is a string with $t$ vertices, $t \geq 1, \mathcal{D}_{2}(T)$ is isomorphic to $\delta_{\left(3,1^{t}\right)}$. More generally, one can see that in this case if $t \geq k-1$, then $\mathcal{D}_{k}(T)$ is isomorphic to $\delta_{\left(k+1,1^{+2-k}\right)}$ (for the case $k=1, t=0$, we use the convention that $\widehat{T}$ is the graph consisting of two vertices which are connected by an edge). Unfortunately, we do not know how to generalize Theorem 3.2, or even Theorem 2.2, to this case.

The family of complexes, which, because of the connections to spaces of monic hyperbolic polynomials with multiple roots, appears to us to be of great interest, is 
$\left\{\delta_{\lambda}\right\}_{\lambda \vdash n}$. It is effortless to extend Definition 3.1 to this case, so that one can talk about $\lambda$-fold disconnecting complexes of trees. The difficulty arises from the fact that we do not know of any natural replacement for the complexes $\Delta(G)$.

One way to view Theorems 3.2 and 2.2 is to see them as expressing a kind of duality. The complexes $\delta_{\lambda}$ are given by listing their maximal simplices, while the complexes $\Delta(G)$ are given by listing minimal nonsimplices, which turn out to have dimension 1 . Such a description would have been impossible for the complexes $\delta_{\lambda}$ themselves, since in fact they contain complete skeletons in the dimensions up to roughly half of the number of vertices.

In light of the above, it seems interesting to ask: Can one find simplicial complexes, having some nice combinatorial description in terms of minimal nonsimplices of small dimension, which are homotopy equivalent to complexes $\delta_{\lambda}$ ?

Theorems 3.2 and 2.2 can be thought of as the first step on the path to providing a complete and satisfactory answer to that question.

\section{Acknowledgment}

I thank the referee for the useful suggestions which led to the improvement of this paper.

\section{References}

1. A. Björner, Topological methods, in Handbook of Combinatorics (eds. R. Graham, M. Grötschel and L. Lovász), Elsevier, Amsterdam, 1995, pp. 1819-1872.

2. R. Ehrenborg, Private communication, 2002.

3. D.N. Kozlov, Rational homology of spaces of complex monic polynomials with multiple roots, Preprint, Institute for Advanced Study, 1999, to appear in Mathematika.

4. D.N. Kozlov, Complexes of directed trees, J. Combin. Theory Ser. A 88(1) (1999), 112-122.

5. D.N. Kozlov, Topology of spaces of hyperbolic polynomials and combinatorics of resonances, Israel J. Math. 132 (2002), 189-206.

6. D.N. Kozlov, Resonance category, Preprint 2001, math.CT/0111196.

7. D. Quillen, Homotopy properties of the poset of nontrivial p-subgroups of a group, Adv. in Math. 28 (1978), 101-128.

8. B. Shapiro and V. Welker, Combinatorics and topology of stratifications of the space of monic polynomials with real coefficients, Results Math. 33(3-4) (1998), 338-355.

9. R.P. Stanley, Private communication, 1996.

Received May 8, 2002, and in revised form November 12, 2003. Online publication May 24, 2004. 